\title{
Entropy Generation Minimization of a Thermoelectric Cooler
}

\author{
F. Gutiérrez and F. Méndez* \\ Departamento de Termofluidos, Facultad de Ingeniería UNAM, 04510 Mexico
}

\begin{abstract}
In the present work, we develop a theoretical analysis to minimize the entropy generation of a thermo-electric cooler (TEC), which can be used to cool a processor. We have applied an analysis of first and second law of the Thermodynamics to a TEC. We consider the entropy generation equation as the objective function and the first law as a restriction, in accordance with the variational calculus theory. The numerical predictions show that minimum entropy generation and the better performance coefficients (COPs) are achieved with the largest figure of merit, which represents a relationship between the Peltier effect (cooling) and the Joule effect (heating).
\end{abstract}

\section{INTRODUCTION}

Thermoelectric cooling allows changing directly electricity into refrigeration. In 1834 Peltier noticed, that when he applied electric current to a circuit, which was made with the junction of two distinct metals, one of the junctions was heated and the other was cooled. Thermoelectric cooling is one application of thermoelectricity; it is the set of phenomena related with processing electricity into heat and vice versa. When an electric current circulates through one or more semiconductors pairs type $n$ and $p$, it produces a temperature gradient between the junctions. It is important to note here that a p-type semiconductor represents a type of semiconductor in which current passes through a solid via electron flow into positive 'holes' in a crystal. The holes are introduced using electron-deficient impurity atoms (doped crystal). Meanwhile an n-type semiconductor is obtained by carrying out a process of doping, that is, by adding an impurity of valence-five elements to a valence-four semiconductor in order to increase the number of free (in this case negative) charge carriers. Energy crosses easily from the low temperature reservoir to the high temperature reservoir through the semiconductor type $n$, meanwhile energy flows hardly through material $\mathrm{p}$ from high temperature to low temperature reservoir [1]. A typical device showing the above operation is represented in Fig. 1.

\section{Thermoelectric Studies}

Nowadays, it is very well-known that an increment in the capacity and reduction size of electronic equipment produces higher heat transfer rates than before $[2,3]$. Phelan and others [4] made a review study about different and nonconventional refrigeration systems; concluding that only TECs can meet the requirements imposed by the electronic miniaturization and to be commercially available.

Omer and others [5] proposed an experimental design, in which a phase change of a material was used in combination

\footnotetext{
*Address correspondence to this author at the Departamento de Termofluidos, Facultad de Ingeniería, Universidad Nacional Autónoma de México, 04510, México D. F., MEXICO; Tel: 52-55-56228103; Fax: 52-5556228106; E-mail: fmendez@servidor.unam.mx
}

with one thermosyphon in order to that the system achieves a refrigeration capacity between 150 and $200 \mathrm{~W}$. Cheng and Shih [6] optimized a set of TECs coupled in cascade using genetic algorithms. Gordon and others [7] coupled a thermoelectric refrigerator with an absorption system; they achieved higher CPOs than those attained by the systems individually.

Yang and others [8] reviewed the performance of TECs employing electric currents varying with time and with values higher to the recommended. Vikhor and Anatychuk [9] used segmented semiconductors $\mathrm{n}$ and $\mathrm{p}$ elements, and they gotten a better performance of TEC, because they changed discreetly TEC properties. Cheng and Lin [10] optimized a TEC geometrically and took the volume used by TEC as a restriction, solving also the problem with genetic algorithms. They got the best cooling capacities when they reduced the characteristic length of semiconductors materials.

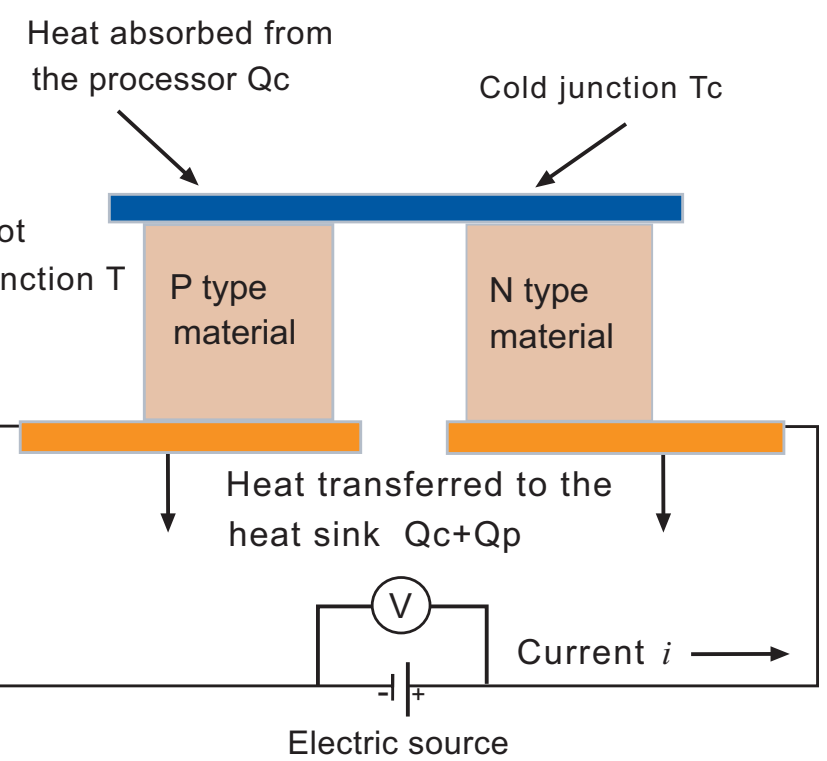

Fig. (1). Outline of a thermoelectric cooler (TEC) device.

In the present work, we have developed a theoretical analysis based on minimization of the entropy generation to 
describe the basic characteristics of a thermoelectric cooler. In particular, the main parameter of refrigeration systems, $i$. e., the performance coefficient (COP) is optimized as a function of the involved dimensionless parameters. We justify the present analysis because the optimization studies based on thermodynamic analysis have received considerable attention in recent years, [11].

\section{Basic Characteristics of a Thermoelectric Cooler (TEC)}

These electric equipments can change directly electric power in a thermal gradient. They are very reliable, silent, small and light. TECs can be reduced to very small scales and do not loss efficiency as it is common in refrigeration systems. On the other hand, the durability is very high because they can work more than $100000 \mathrm{~h}$ [1].

The direction of heat transfer is totally reversible and just depends on the direction of electric current. Thermoelectric equipments allow supporting a precise control of temperature in systems, between $\pm 0.1^{\circ} \mathrm{C}$, with the help of an electronic device suitable. They are used in harmful environments or inside of small places for conventional refrigeration and can work independently from the direction of gravity.

The figure of merit $(Z)$ is one of the most important parameters that describe the performance of TEC; it is defined as the quotient $S^{2} /(r \lambda)$, where $S$ is the Seebeck coefficient, $r$ is the electric resistivity and $\lambda$ is the thermal conductivity.

\section{Entropy Generation}

Thermodynamics analysis has three worthy foundations which basically are described by mass conservation equation, and the first and second law of thermodynamics. The conservation of mass law explains the interactions of mass inside of a system and the first law clarifies the exchanges of energy, the second law gives a quantitative relation of the irreversibility of a system through entropy generation.

Entropy generation, here denoted by the symbol $\dot{S}_{g e n}$, is a very important concept because it is related directly with the lost work ( $\dot{W}_{\text {lost }}$ ) of the system. The Gouy-Stodola theorem establishes that lost work and entropy generation are directly proportional through the relationship:

$$
\dot{W}_{\text {lost }}=T_{0} \dot{S}_{\text {gen }}
$$

where $T_{0}$ is a reference or environment temperature $[11,12]$. On the other hand, the following expression relates the irreversibility of a system with the actual work and with the ideal work:

$$
\dot{W}=\dot{W}_{\max }-\dot{W}_{\text {lost }},
$$

equations (1) and (2) show clearly that when entropy generation is minimum, the actual work $W$ reaches maximum values.

\section{PROBLEM DESCRIPTION}

The present physical system is composed by a thermoelectric cooler (TEC); this device transfers the heat generated by a processor to a heat sink, so this study emphasizes the physical performance of a TEC.

\section{First law of Thermodynamics Analysis}

In order to improve the performance of a TEC applied to cool a processor or chip, we apply an energy balance for the system sketched in Fig. (2).

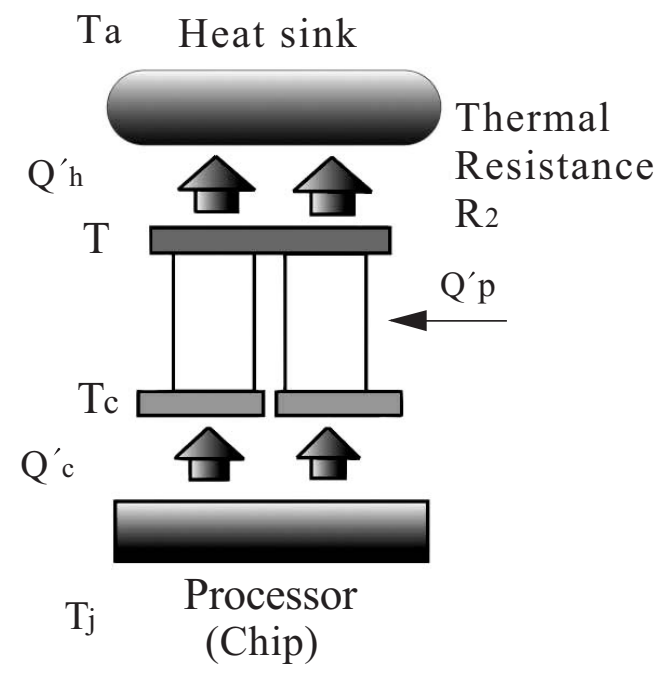

Fig. (2). Schematic diagram of a TEC.

From the above figure, we can appreciate that $\dot{Q}_{c}$ is the heat transfer rate from the processor, $Q_{p}$ is the external power used by the TEC to work continuously, $\dot{Q}_{h}$ is the heat transfer rate dissipated in the heat sink. In addition, $T_{a}$ is the ambient temperature, $T$ the temperature at the hot side of TEC, $T_{c}$ the temperature at the cold side of TEC and $T_{j}$ the temperature of the processor or chip. $\mathrm{R}_{2}$ is the thermal resistance between the ambient and hot side of TEC temperature reservoirs.

Therefore, the use of the first law of thermodynamics allows us distinguish the energy interactions that take place in the TEC and for this case, considering the previous discussion can be written as,

$$
\dot{Q}_{c}+\dot{Q}_{p}-\dot{Q}_{h}=\frac{d E}{d t},
$$

where $\frac{d E}{d t}$ represents the accumulation energy term of TEC.

Explicit relationships for each heat transfer rates are given lines below considering the following: the Peltier effect absorbs energy from the cold junction or reservoir of the processor; however, together with this effect come another two unfavorable effects: the Joule effect and heat conduction. Therefore, we use a relationship for $\dot{Q}_{c}$ [13], which considers the inclusion of the above effects and given by:

$$
Q_{c}=2 N\left[S I T_{c}-\frac{I^{2} r}{2 G}-\lambda G\left(T-T_{c}\right)\right] \text {. }
$$




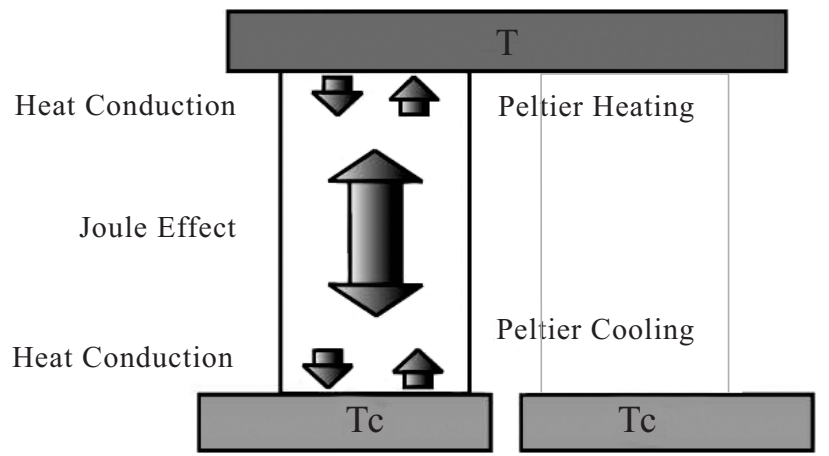

Fig. (3). Heat transfer interactions in a TEC.

In Fig. (3) are represented schematically these effects. The first term of eq. (4) is the Peltier effect, the second is the Joule effect and the third are heat conduction losses. Besides, $N$ is the number of semiconductors materials $n$ and $p, S$ is the Seebeck coefficient, $I$ is the electric current used by TEC to work continuously, $r$ is the electric resistivity, $G$ is a geometric factor from semiconductor materials $(G=$ Area/Length) and $\lambda$ is the thermal conductivity of the thermoelectric element. On the other hand, the expression to calculate the electrical power used by the TEC to run continuously $\dot{Q}_{p}$ is [13]:

$$
Q_{p}=2 N\left[\frac{I^{2} r}{G}+S I\left(T-T_{c}\right)\right],
$$

where the first term in the above relationship corresponds to the Joule effect and the second is the Peltier effect between the temperatures $T$ and $T_{c}$. The expression $\frac{I r}{G}+S\left(T-T_{c}\right)$ is obtained from eq. (5) when we extract an electric current factor $I$ from it. This expression has voltage units and it shows clearly the thermal and electric character of the thermoelectric systems; because the first term represents the voltage due to electric phenomena (Joule effect) and the second is due to temperature gradient (Peltier effect). The expression for the heat transfer rate at the hot side of the TEC can be written as [10]:

$$
Q_{h}=2 N\left[S I T+\frac{I^{2} r}{2 G}-\lambda G\left(T-T_{c}\right)\right],
$$

or equivalently using Figure (2):

$$
\dot{Q}_{h}=\frac{T-T_{a}}{R_{2}} .
$$

This last expression relates the heat transfer rate at the hot side of the TEC with the heat sink. On the other hand, the term $\frac{d E}{d t}$ represents the energy change rate, like internal, kinetic or potential energy. We have approximated the energy $E$ mainly with the internal energy $U$ as $\rho V C T$; where $\rho$ is density, $V$ volume and $C$ specific heat. Therefore, the energy change rate can be written as,

$$
\frac{d E}{d t}=\rho V C \frac{d T}{d t} .
$$

Substituting equations (4)-(8) into eq. (3), considering a uniform electric current and recognizing that the expressions of Peltier and Joule effects and the conduction heat have their origin in constitutive relations; eq. (3) is then,

$$
\begin{aligned}
& 2 N\left[S I T_{c}-\frac{I^{2} r}{2 G}-\lambda G\left(T-T_{c}\right)\right]+ \\
& 2 N\left[\frac{I^{2} r}{G}+S I\left(T-T_{c}\right)\right]-\frac{T-T_{a}}{R_{2}} \\
& =\rho V C \frac{d T}{d t} .
\end{aligned}
$$

\section{Dimensionless Equations}

In order to reduce the number of physical parameters, we can introduce the following dimensionless variables $\theta, \tau$ and $i$ as:

$$
\begin{aligned}
& \theta=\frac{T-T_{c}}{T_{a}-T_{c}} ; \\
& \tau=\frac{t}{t_{0}} ; \\
& i=\frac{I}{I_{0}},
\end{aligned}
$$

where $t_{0}$ and $I_{0}$ are a reference time and electric current respectively, which are to be defined later. Substituting these adimensional variables (eqs. 10a-10c) into the energy balance (eq. 9); we obtain that,

$$
\begin{aligned}
& i \theta+\frac{r I_{0} i^{2}}{S G\left(T_{a}-T_{c}\right)}+\frac{T_{c}}{T_{a}-T_{c}}[i \\
& \left.-\frac{1}{2} \frac{r I_{0} i^{2}}{S G T_{c}}-\frac{\lambda G}{S I_{0}} \frac{T_{a}-T_{c}}{T_{c}} \theta\right] \\
& +\frac{1-\theta}{2 N S I_{0} R_{2}}=\frac{\rho V C}{2 N S I_{0} t_{0}} \frac{d \theta}{d \tau} .
\end{aligned}
$$

From the second term of the left-hand side of the equation 11, and from the right-hand side of the same equation, we can define the reference or characteristic electrical current $I_{0}$ and the reference time $t_{0}$ as: 


$$
I_{0}=\frac{2 G S\left(T_{a}-T_{c}\right)}{r} ;
$$

and

$$
t_{0}=\frac{\rho V C}{2 N S I_{0}}
$$

respectively. Now, we can replace these reference parameters (eqs. 12a, 12b) into the first law (eq. 11), simplifying it in the following relationship,

$$
\begin{aligned}
& i \theta+i^{2}+\frac{T_{c}}{T_{a}-T_{c}} i-\frac{\lambda r}{2 S^{2}\left(T_{a}-T_{c}\right)} \theta \\
& +\left(\frac{r}{4 N G S^{2} R_{2}\left(T_{a}-T_{c}\right)}\right)(1-\theta)=\frac{\mathrm{d} \theta}{\mathrm{d} \tau} .
\end{aligned}
$$

In addition, from this expression (eq. 13), we define the following nondimensional groups:

$$
\begin{aligned}
& \alpha=\frac{\lambda r}{2 S^{2}\left(T_{a}-T_{c}\right)} ; \\
& \beta=\frac{T_{c}}{T_{a}-T_{c}} ; \\
& \varepsilon=\frac{r}{4 N G S^{2} R_{2}\left(T_{a}-T_{c}\right)} .
\end{aligned}
$$

$\alpha$ group clusters the main properties from TEC and its definition is similar to the inverse of figure of merit $\left(\lambda r / S^{2}\right)$. $\beta$ group is a nondimensional temperature parameter and $\varepsilon$ introduces the performance of the high temperature sink.

Replacing these nondimensional expressions (eq. 14) into the first law (eq. 13) and making some simplifications, we can obtain that

$$
(i-\alpha-\varepsilon) \theta+i^{2}+\beta i+\varepsilon=\frac{d \theta}{d \tau} .
$$

\section{Second Law Analysis}

The heat transfer coming from the processor and the heat sent to the heat sink have a strong influence on the entropy generation. Keeping in mind these two energy interactions, the production of entropy takes the following form [11]:

$$
S_{g}=\int_{0}^{t_{0}}\left\{-\frac{\dot{Q}_{c}}{T_{c}}+\frac{\dot{Q}_{h}}{T}\right\} d t .
$$

Substituting the heat transfer interactions defined previously (eqs. 4 and 6 ) into the entropy generation expression, it takes the following format:

$$
\begin{aligned}
& S_{g}=\int_{0}^{t_{0}}\left\{-2 N\left[S I-\frac{I^{2} r}{2 G T_{c}}-\frac{\lambda G}{T_{c}}\left(T-T_{c}\right)\right]\right. \\
& \left.+2 N\left[S I+\frac{I^{2} r}{2 G T}-\frac{\lambda G}{T}\left(T-T_{c}\right)\right]\right\} d t .
\end{aligned}
$$

Defining the non-dimensional entropy generation expression as:

$$
\Phi=\frac{S_{g}}{S_{0}},
$$

where $S_{0}$ is the reference entropy generation that will be defined later. Now substituting the entropy generation eq. 17 in the eq. 18 , we obtain

$$
\begin{aligned}
& S_{0} \Phi=\int_{0}^{t_{0}}\left\{-2 N\left[S I-\frac{I^{2} r}{2 G T_{c}}-\frac{\lambda G}{T_{c}}\left(T-T_{c}\right)\right]\right. \\
& \left.+2 N\left[S I+\frac{I^{2} r}{2 G T}-\frac{\lambda G}{T}\left(T-T_{c}\right)\right]\right\} d t,
\end{aligned}
$$

And employing the nondimensional variables (eqs. 10) into the entropy generation, it can be expressed as:

$$
\begin{aligned}
& S_{0} \Phi=t_{0} \int_{0}^{1}\left\{2 N \left[\frac{I_{0}^{2} r}{2 G T_{c}} i^{2}\right.\right. \\
& \left.+\frac{\lambda G\left(T_{a}-T_{c}\right)}{T_{c}} \theta\right] \\
& +2 N\left[\frac{1}{2} \frac{I_{0}^{2} r}{G\left[T_{c}+\left(T_{a}-T_{c}\right) \theta\right]^{i^{2}}}\right. \\
& \left.\left.-\frac{\lambda G\left(T_{a}-T_{c}\right) \theta}{T_{c}+\left(T_{a}-T_{c}\right) \theta}\right]\right\} d \tau .
\end{aligned}
$$

Substituting the definition of the reference electrical current (eq. 12a) in the above equation (eq. 20) and using the nondimensional groups (eqs. 14), the entropy generation can be simplified to the following relationship,

$$
\begin{aligned}
& \frac{S_{0}}{2 N G \lambda t_{0}} \Phi=\frac{1}{\alpha \beta} \int_{0}^{1}\left\{i^{2}\left(1+\frac{\beta}{\beta+\theta}\right)\right. \\
& \left.+\alpha \beta\left(\frac{\theta}{\beta}-\frac{\theta}{\beta+\theta}\right)\right\} d \tau .
\end{aligned}
$$

From the left-hand side of equation (21), we define the reference entropy generation as: 


$$
S_{0}=\frac{2 N G \lambda t_{0}}{\alpha \beta}
$$

And using the definitions of $t_{0}$ (eq. 12b) and $\alpha$ (eq. 14a), the reference entropy generation can be rewritten as:

$$
S_{0}=\frac{\rho V C}{\beta},
$$

with the above definition, the dimensionless entropy generation simplifies considerably and can be rewritten through the following relationship,

$$
\begin{aligned}
& \Phi=\int_{0}^{1}\left\{i^{2}\left(1+\frac{\beta}{\beta+\theta}\right)\right. \\
& \left.+\alpha \beta\left(\frac{\theta}{\beta}-\frac{\theta}{\beta+\theta}\right)\right\} d \tau,
\end{aligned}
$$

\section{Optimization}

With the objective of reducing the non-dimensional entropy generation within an operation period, we can minimize it through the use of variational calculus. We consider the nondimensional entropy generation (eq. 24) as the functional expression and the adimensional energy balance or first law (eq. 15) as a constraint in accordance with theory of variational calculus [14]. We can appreciate that the factor $\alpha \theta$ appears in the second term of the entropy generation equation and in the first term of the energy conservation equation; therefore, we can combine both equations in only one equation

$$
\begin{aligned}
& \Phi=\int_{0}^{1}\left\{i^{2}\left(1+\frac{\beta}{\beta+\theta}\right)+(i-\varepsilon) \theta+i^{2}\right. \\
& \left.+\beta i+\varepsilon-\frac{d \theta}{d \tau}-\frac{\alpha \beta \theta}{\beta+\theta}\right\} d \tau
\end{aligned}
$$

and in order to solve this functional, we need to solve the following Euler-Lagrange equation (Rao WILEY 1996) [14]:

$$
\frac{\partial F}{\partial \theta}-\frac{d}{d \tau}\left(\frac{\partial F}{\partial \dot{\theta}}\right)=0
$$

where the functional $F$ is the argument of the previous integral (eq. 25); applying therefore this formulation, the resulting equation can be written as

$$
-\frac{\beta i^{2}}{(\beta+\theta)^{2}}+\mathrm{i}-\varepsilon-\frac{\alpha \beta^{2}}{(\beta+\theta)^{2}}=0,
$$

which can readily solve for $\theta$ given that

$$
\theta=\sqrt{\frac{\beta\left(i^{2}+\alpha \beta\right)}{i-\varepsilon}}-\beta
$$

Here, we only consider the positive roots of the term $\sqrt{\frac{\beta\left(i^{2}+\alpha \beta\right)}{i-\varepsilon}} \cdot$ Obviously, we assume that the other solution given by $\theta=-\sqrt{\frac{\beta\left(i^{2}+\alpha \beta\right)}{i-\varepsilon}}-\beta$ has not a physical sense because in this case the physical temperature of the system $T$ would be lower than temperature $T_{c}$, which is impossible to occur.

This expression does not depend on time, then the nondimensional entropy generation (eq. 25) can be solved directly. The corresponding solution, in terms of $\theta$, is given by

$$
\begin{aligned}
& \Phi=i^{2}\left(1+\frac{\beta}{\beta+\theta}\right)+(i-\varepsilon) \theta+i^{2} \\
& +\beta i+\varepsilon-\frac{d \theta}{d \tau}-\frac{\alpha \beta \theta}{\beta+\theta}
\end{aligned}
$$

\section{Nondimensional Heat Transfer}

We define the nondimensional heat transfer used to cooling the processor as:

$$
\varphi_{c}=\frac{\dot{Q}_{c}}{\dot{Q}_{0}}
$$

where $\dot{Q}_{0}$ is a reference heat transfer that will be defined later. Using the definition of the cooling heat transfer (eq. 4) and substituting it into the adimensional expression (eq. 30), we obtain that

$$
\varphi_{c}=\frac{2 N}{\dot{Q}_{0}}\left[S I T_{c}-\frac{I^{2} r}{2 G}-\lambda G\left(T-T_{c}\right)\right] \text {; }
$$

taking into account the definition of the nondimensional variables $\theta$ and $i$ (eqs. 10a and 10c, respectively) and substituting them in the adimensional cooling heat transfer (eq. 31), we obtain

$$
\begin{aligned}
& \varphi_{c}=\frac{2 N}{Q_{0}}\left[S I_{0} T_{c} i-\frac{I_{0}^{2} r}{2 G} i^{2}\right. \\
& \left.-\lambda G\left(T_{a}-T_{c}\right) \theta\right],
\end{aligned}
$$

by means of the nondimensional groups $\alpha, \beta$ and the reference electrical current $I_{0}$, the expression (32) can write as,

$$
\begin{aligned}
& \varphi_{c}=\frac{4 N G S^{2}\left(T_{a}-T_{c}\right) T_{c}}{Q_{0} r}[i \\
& \left.-\frac{i^{2}}{\beta}-\frac{\alpha}{\beta} \theta\right] .
\end{aligned}
$$


In addition, we define the reference heat transfer $\dot{Q}_{0}$ as:

$$
Q_{0}=\frac{4 N G S^{2}\left(T_{a}-T_{c}\right) T_{c}}{r} ;
$$

Therefore, the nondimensional expression for the cooling heat transfer is:

$$
\varphi_{c}=i-\frac{i^{2}}{\beta}-\frac{\alpha}{\beta} \theta .
$$

In a similar way, we can define the nondimensional power used by the TEC as:

$$
\varphi_{p}=\frac{Q_{p}}{Q_{0}},
$$

Using the expression for the power used by the TEC (eq. 5) into equation (36):

$$
\begin{aligned}
& \varphi_{p}=\frac{r}{2 G S^{2}\left(T_{a}-T_{c}\right) T_{c}}\left[\frac{I^{2} r}{G}\right. \\
& \left.+S I\left(T-T_{c}\right)\right] .
\end{aligned}
$$

Through the nondimensional groups $\alpha, \beta$ and the reference electrical current, the nondimensional power input can readily derive and write as:

$$
\varphi_{p}=\frac{1}{\beta}\left[i \theta+2 i^{2}\right] ;
$$

employing $\varphi_{c}$ and $\varphi_{p}$ the COP can be expressed as:

$$
C O P=\frac{\varphi_{c}}{\varphi_{p}},
$$

which is equivalent to write,

$$
C O P=\frac{\beta i-i^{2}-\alpha \theta}{i \theta+2 i^{2}} .
$$

On the other hand, the nondimensional entropy generation integrated over a period does not depend on time $\tau$, so we can generate a set of graphics of the nondimensional dependent variables as functions only of the groups or dimensionless parameters. However, we can simplify the definition of the nondimensional entropy generation (combining eqs. 18, 23) and writes it as:

$$
\Phi=\frac{S_{g} \beta}{\rho V C} .
$$

Then, we propose a new reference entropy generation as:

$$
S_{0 N}=\rho V C,
$$

and the adimensional entropy generation should be written as

$$
\Phi_{\beta}=\frac{S_{g}}{S_{0 N}},
$$

or, the new dimensionless entropy generation can be written by means of the following relationship,

$$
\Phi_{\beta}=\frac{\Phi}{\beta} \text {. }
$$

\section{RESULTS}

In this section, we present the distributions of the nondimensional temperature $\theta$ (eq. 28), cooling heat transfer $\varphi_{c}$ (eq. 32), electric power $\varphi_{p}$ (eq. 38), $C O P$ (eq. 40) and entropy generation $\Phi_{\beta}$ (eq. 44) as functions of the nondimensional parameters. In all figures, we have used data reported by Chein and Huang [13] and the TECs manufacturer Melcor [15]. The data is: $N=301, S=0.0002 \mathrm{~V} / \mathrm{K}, r=0.00001 \mathrm{~m}$, $\lambda=0.15 \mathrm{Wm}^{-1} \mathrm{~K}^{-1}, G=0.1196 \mathrm{~m}$. Besides, the conductivity value is one order lower than indicated in surveys and the geometric factor is one order higher. These values correspond to the pink curve.

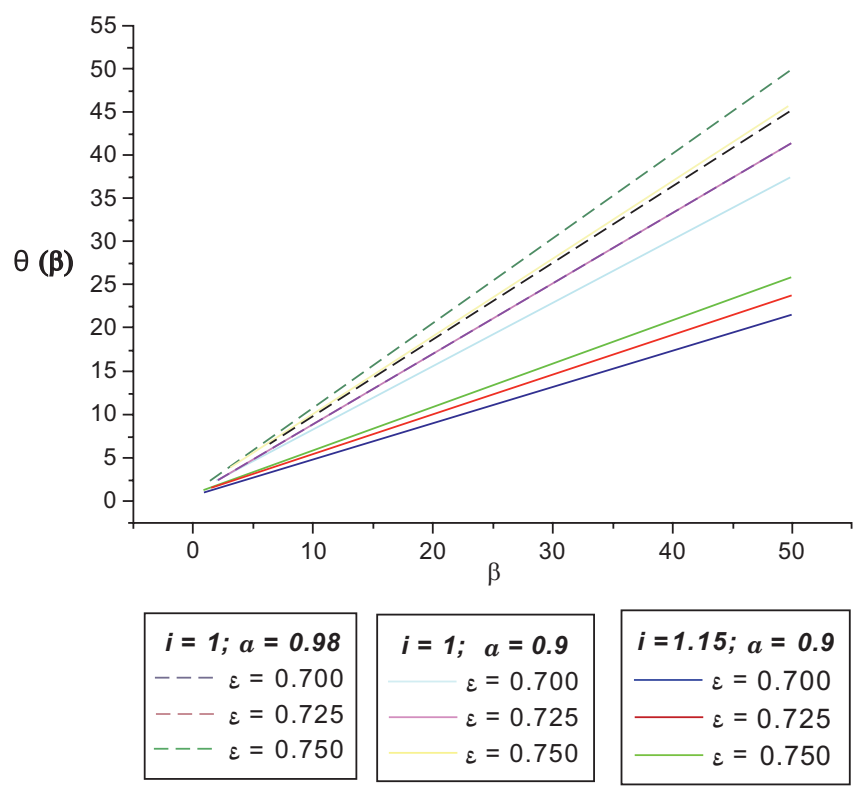

Fig. (4). Nondimensional temperature $\theta$ as a function of the nondimensional parameters.

The shape of $\theta$ (Fig. 4) is almost a straight line within the domain of the used parameters. This means that the figure of merit parameter, $S^{2} / \lambda r$, has to be as higher as can be possible to achieve a lower temperature $T$ in the hot side of the TEC. This condition reduces the requirements in the heat sink. For the same reason is convenient that the adimensional current becomes higher than one.

The shape of $\varphi_{c}$ (Fig. 5) is asymptotic to $\beta$ large. This profile is the result of the competition between the Peltier and Joule effects and heat conduction, where the last two are dissipative effects. A large surface and a small length of the thermoelectric cooler elements yields an increase in the useful cooling power, because $G=$ Area/Length; this result is the 
same as that expressed by other authors like Simons and Chu [3] or Cheng y Lin [10].

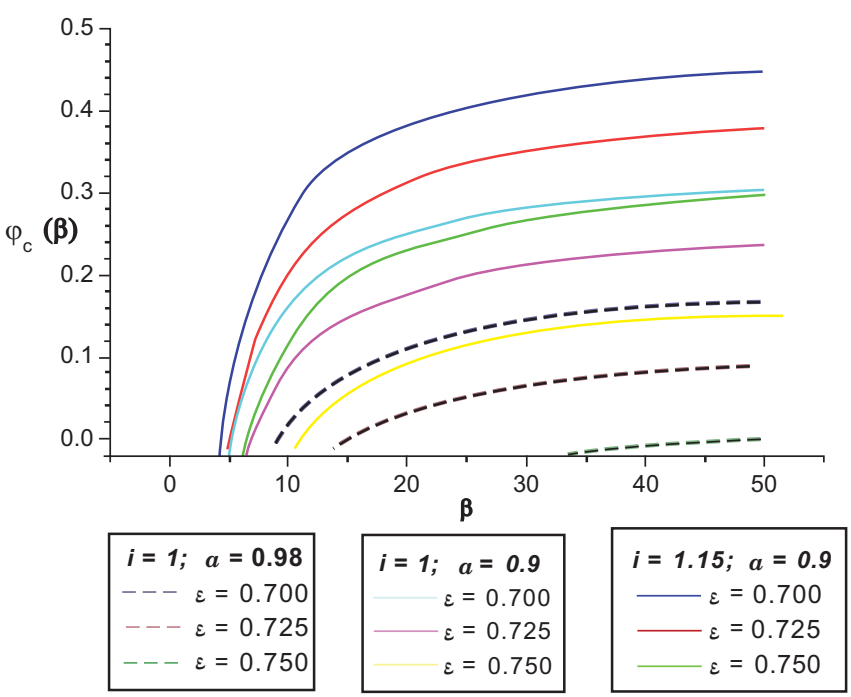

Fig. (5). Cooling heat transfer $\varphi_{c}$ as a function of the nondimensional parameters.

The growing behavior of $\varphi_{c}$ with $\beta$ means that when the temperature at the cold side of TEC is very low the amount of cooling heat $\varphi_{c}$ decreases, according to the definition of $\beta$ (Fig. 6); this behavior is similar to that shown by common refrigeration systems. However, when the temperature difference $T_{a}-T_{c}$ is close to zero, the amount of heat cooling capacity increases.

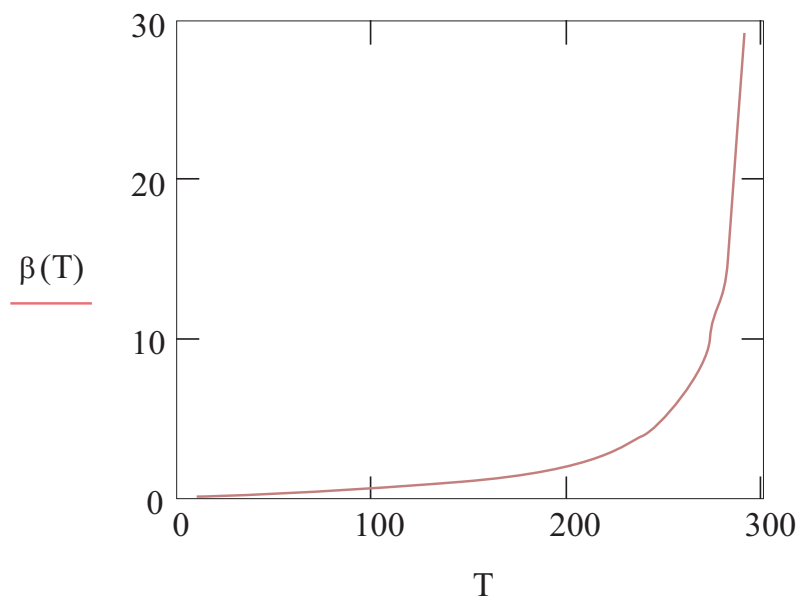

Fig. (6). Graphic of $\beta$ as a function of nondimensional parameters and with $\mathrm{T}_{\mathrm{a}}=300 \mathrm{~K}$.

According to the definitions of the adimensional variables and parameters (eqs. 10, 12 and 14), we achieve large values of $\alpha$ with small values of the figure of merit $S^{2} / r \lambda$, $\varepsilon$ can have arbitrary values, but among them when the factor $4 N G R_{2}$ is large, we get lower values of $\varepsilon$, this means that the cooling capacity increases when the number of thermo electric pairs, area of elements and high temperature sink are large or the length of elements is short. The amount of electric current has a direct impact in the performance of TEC, because the electric current $I$ is higher than the reference electric current $I_{0}$ the cooling heat transfer increase. The best performance is shown in Fig. (5) by the curve with $\alpha=0.9, \varepsilon$ $=0.7$ and $i=1.15$ (solid blue).

The downward trend in $\varphi_{p}$ (Fig. 7) indicates that when the $T_{a}-T_{c}$ increases, the required input power $\frac{\dot{Q}_{p}}{A}$ does it also. We obtain the lower useful power with smaller values of $\varepsilon$. Again larger figure of merit are preferred with $i>1$.

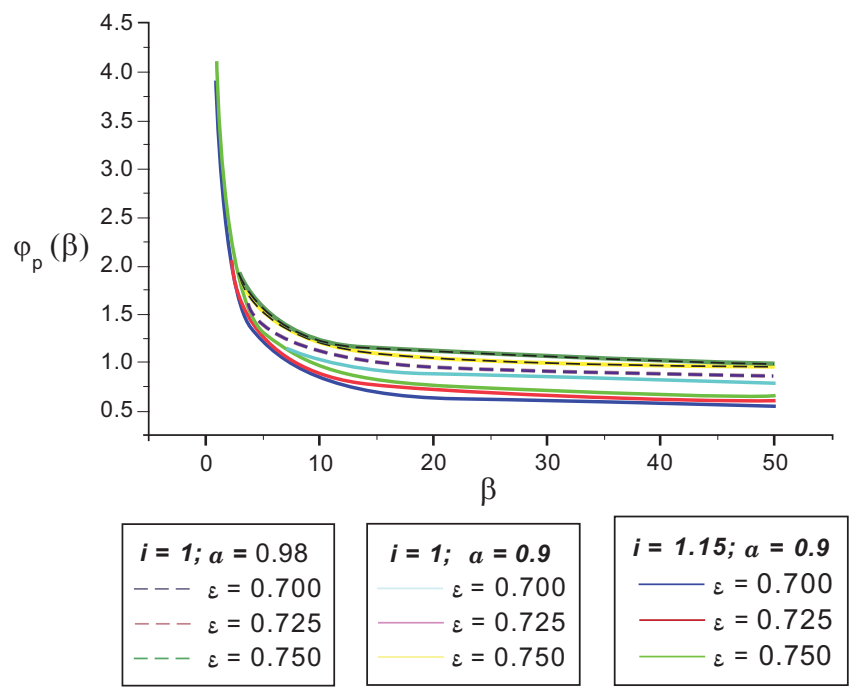

Fig. (7). Non dimensional power input $\varphi_{p}$ as a function of the nondimensional parameters.

The growing trend of COP (Fig. 8) shows that by decreasing $T_{a}-T_{c}$ is obtained a better performance of TEC. This result is similar to that of Cheng and Lin [10], whom mentioned that by requiring a greater cooling temperature difference COP falls, and the betters COPs are those with values of $\alpha$ and $i$ large and $\varepsilon$ small.

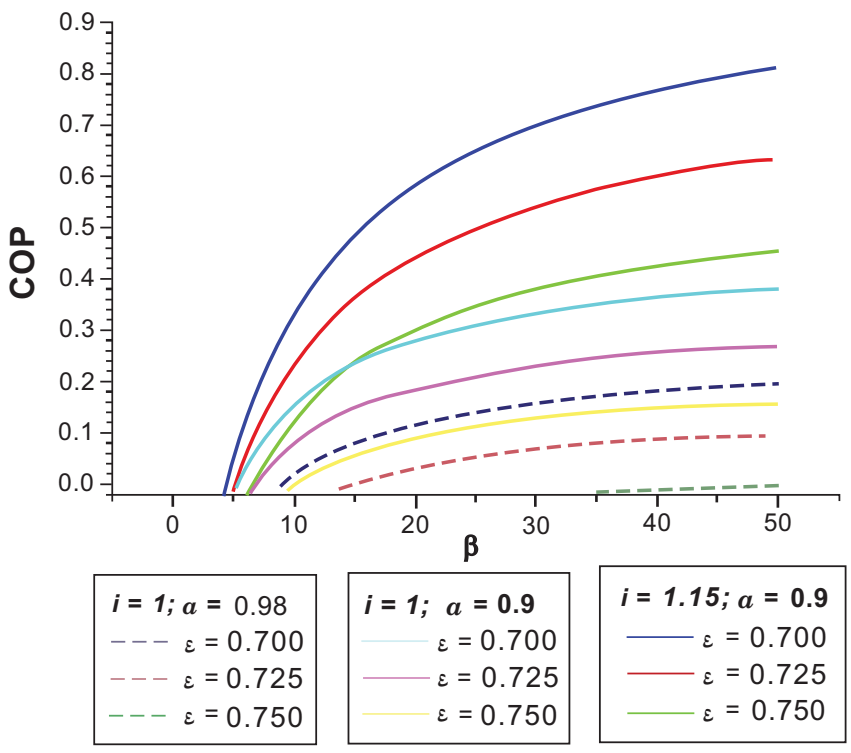

Fig. (8). COP as a function of the nondimensional parameters.

The shape of $\Phi_{\beta}$ is decreasing with $\varepsilon$ (Fig. 9); physically this means that the entropy generation decrease when the difference $T_{a}-T_{c}$ is small. 
Therefore, it is desirable a great $4 N G R_{2}$ factor, small values of $\alpha$, which are gotten with a large figure of merit; finally the lowers values of $\Phi_{\beta}$ are achieved when $i=1.15$.

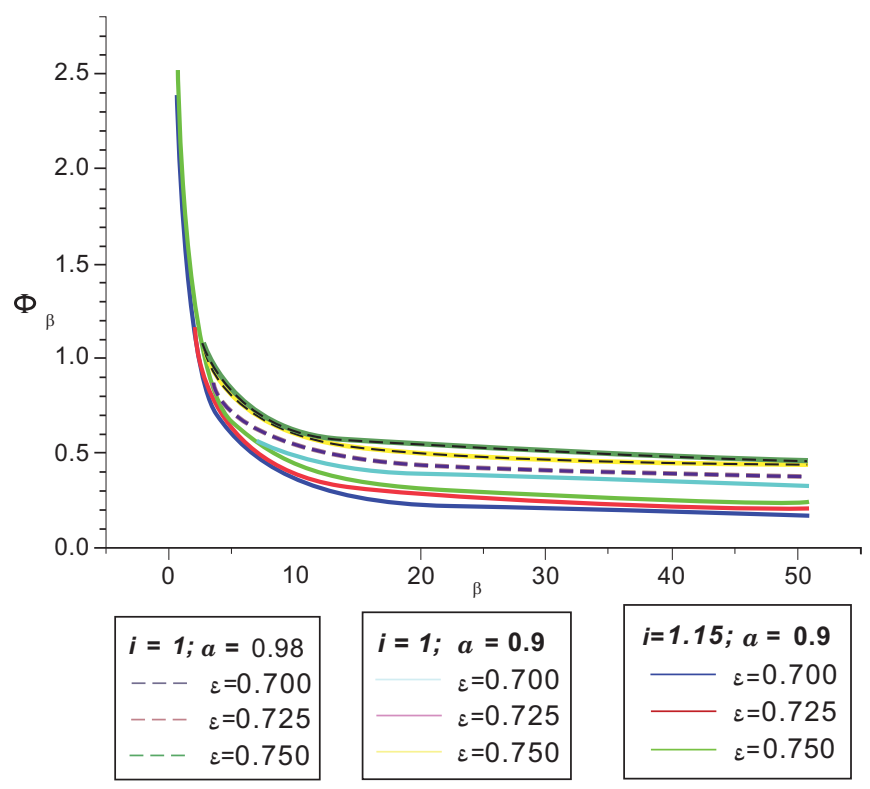

Fig. (9). Non dimensional entropy generation $\Phi_{\beta}$.

The above behavior of the nondimensional entropy generation $\Phi_{\beta}$ is similar to that presented by Bejan in a thermal machine located between two thermal reservoirs [11]. The interaction of the TEC inside the system has three main temperature reservoirs; these are the temperature from the cold side of the TEC, the ambient temperature and the temperature from the heat sink.

The heat transfer interactions are pictured schematically in Figs (10 and 11). These two diagrams depict the whole heat transport phenomena. At the beginning, the heat is "pumped" from the cold side of the TEC to the high temperature sink (Fig. 10), where it is dissipated from this temperature to the ambient temperature (Fig. 11).

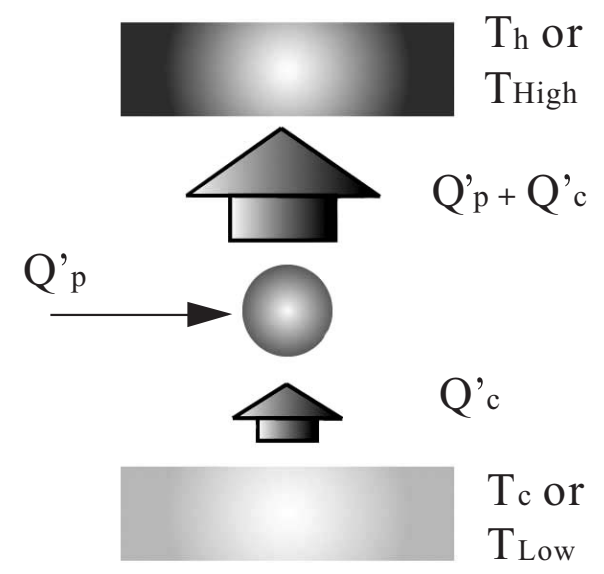

Fig. (10). Heat transfer from the cold side of the TEC to the heat sink.

The entropy generation of these interactions is:

$$
S_{g e n A}=-\frac{\dot{Q}_{c}}{T_{L}}+\frac{\dot{Q}_{c}+\dot{Q}_{p}}{T_{H}},
$$

where, $S_{\text {genA }}$ is the entropy generation of the first stage, according with the reference; besides, $T_{L}$ and $T_{H}$ are the temperatures at the cold side of the TEC and the temperature of the heat sink. $Q_{c}$ and $Q_{p}$ are the same heat transfer interactions defined previously. The heat rejected to the ambient is:

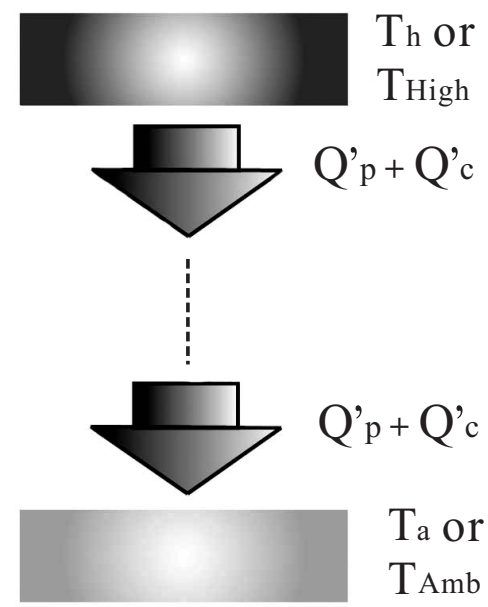

Fig. (11). Heat transfer from the heat sink to the ambient.

The entropy generation produced by this interaction $S_{\text {gen } B}$ is:

$$
S_{g e n B}=-\frac{Q_{c}+Q_{p}}{T_{H}}+\frac{Q_{c}+Q_{p}}{T_{a}}
$$

Finally, the whole entropy generation according to the above reference [11], is the sum:

$$
\sigma=S_{g e n A}+S_{g e n B}
$$

this means that,

$$
\sigma=Q_{c} \frac{T_{a}-T_{L}}{T_{a} T_{L}}+\frac{Q_{p}}{T_{a}} .
$$

This expression is similar to that shown by Bejan for a heat transfer across finite temperature difference. Second term of the right side (eq. 48) represents the entropy generation due to heat generated by the input power, because the heat sink dissipates not only the cooling heat transfer $Q_{c}$ but also the power added to the system in order it works continuously $Q_{p}$.

The shape of the entropy generation $\sigma$ according to the reference (Fig. 12) is similar to the nondimensional entropy generation (Fig. 9). The cooling heat transfer and the power input are unit and the ambient temperature is $300 \mathrm{~K}$.

Different values of $Q_{c}$ or $Q_{p}$ have a little effect in the shape of the curve, they can generate more or less entropy (up or down the curve); however the shape remains. 


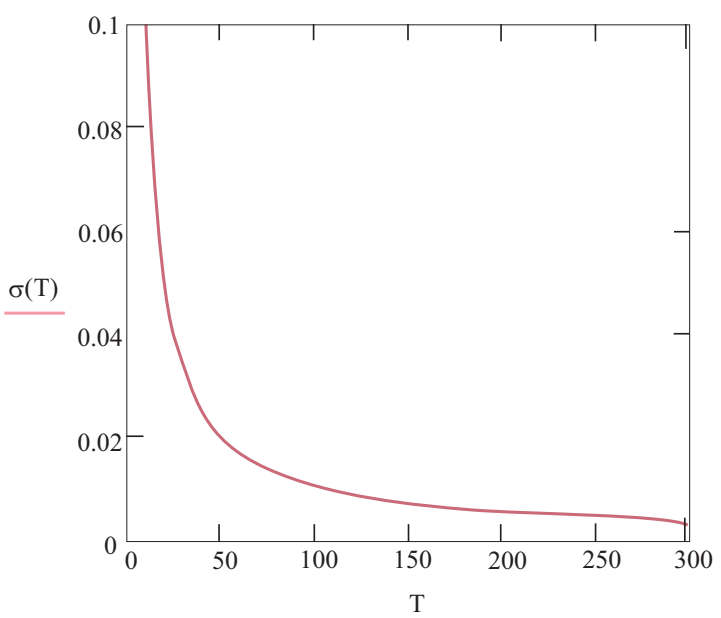

Fig. (12). Entropy generation between a finite temperature difference.

These last two figures are similar, because when we need a larger cooling temperature (temperature at the cold side of TEC) the entropy generation increases, according to the definition of $\beta$ (eq. 14b) and a low temperature $(T)$ corresponds to small values of $\beta$ (Fig. 6). In both figures this situation produces the highest values of generation.

And vice versa when the cold side temperature is similar to ambient temperature, this means higher values of $T$ and $\beta$, the entropy generations ( $\Phi_{\beta}$ and $\sigma$ ) decreases to low values.

\section{CONCLUSIONS}

We used the entropy generation minimization method with the aim of improving the performance of a TEC; whereby we used the first and second law of thermodynamics and variational calculus. The best performance of TEC leads to a greater heat transfer cooling, less useful power consumption and a higher COPs.

We found a dimensionless temperature expression (eq. 28) through variational calculus; this result matches to those works where the entropy generation is minimal. By means of this optimized temperature, we developed the cooling heat transfer $\dot{Q}_{c}$, useful power consumption $\dot{Q}_{p}$, COP and entropy generation $\ddot{O}_{\hat{a}}$ expressions. This set of expressions tells us, which are the best conditions of operation, such that the energy interactions to be as close to a reversible behavior.

Entropy generation minimization $(17,29)$ shows us that the best performance of TEC is achieved with a small $\alpha$ or $\varepsilon$ and employing eq. (11); this means large values of Seebeck coefficient $S$, thermoelectric pairs number $N$, thermal resistance $R_{2}$, geometrical factor $\mathrm{G}$ or with a small electrical resistance $r$, thermal conductivity $\lambda$. Larger geometrical factors are obtained when the area of the thermoelectric element is incremented or when the length is reduced. These conclusions in $\mathrm{G}, \mathrm{N}$ or figure of merit $\left(S^{2} / \lambda r\right)$ are similar to those explained by Phelan and others [4], Chen and Lin [10], and Min and Rowe [16] in their studies.

\section{APPENDIX}

\section{A. Thermo - Electricity}

Thermoelectricity involves different phenomena, which are related with the conversion of heat into electricity and vice versa. There are five main thermoelectric phenomena, and they are Seebeck, Peltier, Thomson, Joule, and heat conduction effects $[17,18]$.

\section{Seebeck Effect}

Seebeck found in 1821, that a temperature difference could be turned into a voltage difference. When an electric circuit of two different metals is heated at one junction, then a temperature difference between the junctions will be produced and this will generate an electro motive force (emf).

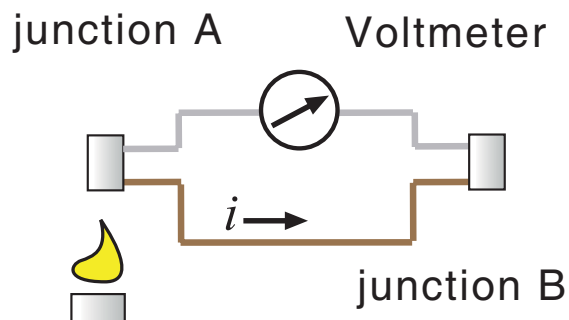

\section{Candle}

Fig. (13). Thermoelectric circuit, Seebeck effect.

The Seebeck effect is due to the electrons difference between the materials and the temperature difference at the junctions.

\section{Peltier Effect}

This effect is opposite of Seebeck, because it produces a temperature difference at the junctions when an emf is applied to the circuit. This effect is reversible, this means, it depends only of the direction of the electric current, (Fig. 14).
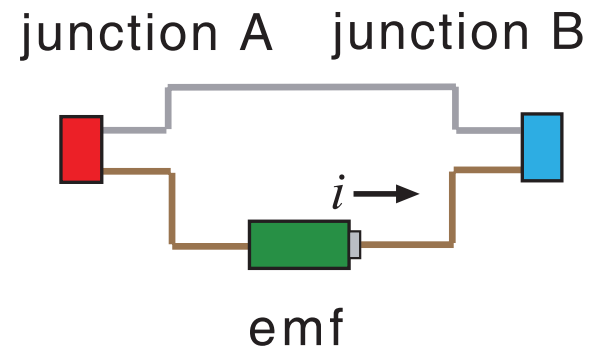

Fig. (14). Thermoelectric circuit, Peltier effect.

\section{Thomson Effect}

It describes the heating or cooling of a conductor at the presence of a temperature gradient, when a current pass through it. When the current moves from the cold junction to the hot, the electrons gain potential energy, and vice versa when they travel from hot to cold junction they lose energy.

If the current passes trough the same conductor the changes in potential energy are canceled; however, when it travels through different conductors, the gradients of potential energy are not necessarily canceled. 


\section{Heat Conduction}

It is due to the heat conduction through the semiconductors materials; from the junction of high temperature to the cold one. Heat conduction is presented both in semiconductor $n$ and $p$. The heat transfer rate is proportional to the temperature gradient.

\section{Joule Effect}

This effect explains the heating of an electric conductor when a current passes through it.

\section{Coupled Heat and Electric Phenomena}

Kelvin, Seebeck, Peltier, and Onsager performed different studies, about the analysis of two simultaneous energy flows through a conductor.

Kelvin proved that the irreversible effects can not be eliminated through the geometric dimensions of the semiconductors materials. He equalized to zero all the entropy changes and deduced some relations, and Onsager confirmed them lately. Onsager developed a study about coupled irreversible flows, Heikes and Ure [17] present a summary about its theory.

\section{B. Variational Calculus}

This theory allows to finding the extreme (maximum or minimum) of a functional. A functional is a function of others functions within a domain. Variational calculus can solve problems of optimal trajectories.

\section{Fundamental Problem of Variational Calculus}

The main objective of the variational calculus is to finding a function $u(x)$ that minimizes the functional (integral):

$$
A=\int_{x_{1}}^{x_{2}} F\left(x, u, u^{\prime}, u^{\prime \prime}\right) d x
$$

where $\mathrm{F}$ is a functional, because it is a function of other functions, and $x$ is the independent variable, then:

$$
\begin{aligned}
& u=u(x) \\
& u^{\prime}=\frac{d u(x)}{d x} ; \\
& u^{\prime \prime}=\frac{d^{2} u(x)}{d x^{2}}
\end{aligned}
$$

Normally, the functional has a physical meaning; in this study is the entropy generation. For other cases, it could be potential energy, a trajectory or the surface of a body.

The integral (eq. 49) is well defined within the domain $\left[x_{1}, x_{2}\right]$, where $\mathrm{u}\left(x_{1}\right)$ and $\mathrm{u}\left(x_{2}\right)$ are the boundary conditions of the problem. The procedure for resolving the problem is to propose a solution and compare it with the exact solution.

The difference between the exact solution and the proposed is minimized, and this can be done in a similar way as in the ordinary calculus [14]. Finally, the minimal difference must satisfy the Euler - Lagrange equation:

$$
\frac{d F}{d x}-\frac{d}{d x}\left(\frac{\partial F}{\partial u^{\prime}}\right)+\frac{d}{d x^{2}}\left(\frac{\partial F}{\partial u^{\prime \prime}}\right)=0 .
$$

When the problem has one or more constraints, the method of Lagrange multipliers can be applied. For example, there is a functional $B$ :

$$
B=\int_{V} f\left(x, y, z, u_{1}, u_{2}, u_{3}, \ldots, u_{n}, \frac{d u}{d x}, \ldots\right) d x
$$

and the $m$ constraints:

$$
\begin{aligned}
& g_{1}\left(x, y, z, u_{1}, u_{2}, u_{3}, \ldots, u_{n}, \frac{d u}{d x}, \ldots\right) \\
& g_{2}\left(x, y, z, u_{1}, u_{2}, u_{3}, \ldots, u_{n}, \frac{d u}{d x}, \ldots\right) \\
& g_{n}\left(x, y, z, u_{1}, u_{2}, u_{3}, \ldots, u_{n}, \frac{d u}{d x}, \ldots\right)
\end{aligned}
$$

Then, based on the Lagrange multipliers, the new functional $C$ to solving is:

$$
C=\int_{V}\left(f+\lambda_{1} g_{1}+\lambda_{2} g_{2}+\ldots+\lambda_{m} g_{m}\right) d x
$$

where $f$ is the argument of the integral $B$ (eq. 52) and $\lambda_{1}$, $\lambda_{2}, \ldots, \lambda_{m}$ are the Lagrange multipliers. To solve this new functional, we can apply the Euler Lagrange equation to the argument of $C$.

$\begin{array}{lll}\text { NOMENCLATURE } \\ C_{l} & =\text { Specific heat } \\ \text { COP } & =\text { Performance coefficient } \\ E & = & \text { Energy } \\ F & = & \text { Arbitrary functional } \\ G & = & \text { Geometric factor } \\ i & = & \text { Dimensionless electric current } \\ I & = & \text { Electrical current } \\ N & = & \text { Heamber of semiconductors } \\ \dot{Q}_{c} & =\text { Dissipated heat transfer rate } \\ \dot{Q}_{h} & =\text { External power } \\ \dot{Q}_{p} & =\text { Electric resistivity } \\ r & \text { Thermal resistance } \\ \mathrm{R}_{2} & =\text { Seebeck coefficient } \\ \dot{S}_{g} & =\text { Entropy generation } \\ \dot{S}_{0} & =\text { Reference entropy generation } \\ t & =\end{array}$




$\begin{array}{lll}T & = & \text { Hot side temperature } \\ T_{a} & = & \text { Ambient temperature } \\ T_{c} & = & \text { Cold side temperature } \\ T_{j} & = & \text { Processor temperature } \\ T_{0} & = & \text { Reference or environment temperature } \\ \text { TEC } & = & \text { Thermo electric cooler } \\ U & = & \text { Internal energy } \\ V & = & \text { Volume } \\ \dot{W}_{1} & =\text { Work } \\ \dot{W}_{\text {lost }} & =\text { Lost work } \\ \dot{W}_{\max } & =\text { Maximum work } \\ Z & =\text { Figure of merit, } S^{2} / r \lambda\end{array}$

\section{Greek letters}

$\begin{array}{lll}\alpha & = & \text { Dimensionless parameter, eq. (14a) } \\ \beta & = & \text { Dimensionless parameter, eq. (14b) } \\ \varepsilon & = & \text { Nondimensionless parameter, eq. (14c) } \\ \Phi & = & \text { Dimensionless entropy } \\ \varphi & = & \text { Dimensionless heat transfer rate (proces- } \\ \varphi & = & \text { Dor) } \\ \lambda & = & \text { Thermal conductivity } \\ \rho & = & \text { Density } \\ \theta & = & \text { Nondimensional temperature } \\ \tau & = & \text { Nondimensional time }\end{array}$

\section{REFERENCES}

[1] S.B. Riffat, and X. Ma, "Thermoelectrics: a review of present and potencial applications", Appl. Therm. Eng., vol. 23, 8, pp. 913-935, June 2003.

[2] C.H. Amon, J. Murthy, S.C. Yao, S. Narumanchi, C.F. Wu, and C.C. Hsieh, "MEMS enabled thermal management of high-heat- flux devices EDIFICE: embedded droplet impingement for integrated cooling of electronics", Exp. Thermal Fluid Sci., vol. 25, 5, pp. 231-242, Nov. 2001.

[3] R.E. Simons, and R.C. Chu, "Application of thermoelectric cooling to electronic equipment: a review and analysis", Sixteenth IEEE Semi-Therm. Symposium 2000.

[4] P.E. Phelan, V.A. Chiriac, and T.Y.T Lee, "Current and future miniature refrigeration cooling technologies for high power microelectronics", IEEE Trans Comp. Pack. Technol., vol. 25, 3, pp. $356-365$, Sep 2002.

[5] S.A. Omer, S.B. Riffat, and X. Ma, "Experimental investigation of a thermoelectric refrigeration system employing a phase change material integrated with thermal diode," Appl. Therm. Energ., vol. 21, 12, pp. 1265-1271, Aug. 2001.

[6] Y.H. Cheng, and C. Shih, "Maximizing the cooling capacity and COP of two-stage thermoelectric coolers through genetic algorithm", Appl. Therm. Eng., vol. 26, 8-9, pp. 937-947, June 2006.

[7] J.M. Gordon, K.C. Ng, H.T. Chua, and A. Chakraborty, "The electro-adsorption chiller: a miniaturized cooling cycle with applications to micro-electronics", Int. J. Refrig., vol. 25, 8, pp. 10251033, Dec. 2002

[8] R. Yang, G. Chen, A.R. Kumar, G.J. Snyder, and J.P. Fleurial, "Transient cooling of thermoelectronic coolers and its applications for microdevices", Energ. Conv. Manag., vol. 46, 9-10, pp. 14071421, June 2005.

[9] L.N. Vikhor, and L.I. Anatychuk, "Theoretical evaluation of maximum temperature difference in segmented thermoelectric coolers", Appl. Therm. Eng., vol. 26, 14-15, pp. 1692-1696, Oct. 2006.

[10] Y.H. Cheng, and W.K. Lin, "Geometric optimization of thermoelectric coolers in a confined volume using genetic algorithms", Appl. Therm. Eng., vol. 25, 17-18, pp. 2983-2997. Dec. 2005.

[11] A. Bejan. "Entropy Generation Minimization”, CRC Press 1996.

[12] M.J. Moran, "Availability analysis: a guide to efficient energy use, corrected edition", ASME Press, New York 1989.

[13] R. Chein, and G. Huang, "Thermoelectric cooler application in electronic cooling", Appl. Therm. Eng., vol. 24, 14-15, pp. 22072217, Oct. 2004.

[14] Rao, S. "Engineering Optimization: Theory and Practice" Third Edition, John Wiley \& Sons, 1996

[15] Melcor. http://www.melcor.com/tec.html, March 28, 2008

[16] G. Min, and D.M. Rowe, "Cooling performance of integrated thermoelectric micro cooler" Solid State Electron., vol. 43, 5, pp. 923-929, May 1999.

[17] R.R. Heikes, and R.W. Ure, "Thermoelectricity: Science and Engineering" Interscience publishers, Chap. 2, 1961.

[18] D.D. Pollock "Physics of Engineering of Materials" Prentice Hall, Chap. 7, 1990

This is an open access article distributed under the terms of the Creative Commons Attribution License (http://creativecommons.org/licenses/by/2.5/), which permits unrestrictive use, distribution, and reproduction in any medium, provided the original work is properly cited. 